\title{
AN AUTOMATIC STEPLENGTH CONTROL ALGORITHM FOR STIFF ODES SYSTEMS
}

\author{
E. A. ORady \\ Industrial and Systems Engineering Department, University of Michigan-Dearborn, \\ 4901 Evergreen Rd, Dearborn, MI 48128, U.S.A.
}

(Received 1 April 1991)

\begin{abstract}
The transient convective-transport heat transfer equation is applicable to many manufacturing processes when analyzing their thermal behavior. The finite element formulation for such a problem on a process domain produces a system of ordinary differerential equations which is characterized to be 'stiff'. Careful handling of the solution is required in order to avoid oscillatory and inaccurate results. This problem can be avoided by selecting a suitable recurrence scheme together with an efficient steplength control algorithm. In this paper an automatic steplength control algorithm has been developed and implemented in a finite element program for the transient convective-transport equation. The algorithm has been tested using an example representing a manufacturing process.
\end{abstract}

\section{INTRODUCTION}

The convective-transport equation is applied to many manufacturing processes to determine the transient temperature distribution [1-7]. It is applied to metal cutting, metal casting, forming, welding, etc. The analytical solution is found to be difficult and often not possible in such applications due to the complexity of the problem [4]. Therefore, numerical methods such as finite difference and finite element are usually employed. The finite element formulation produces a system of first-order ordinary differential equations (ODEs). The system of ODEs is found to be, in most cases, very 'stiff' and produces oscillatory results when the time step is inadequate at the initial stages of the solution $[8,9]$. In order to avoid these problems, there is a need for an automatic time steplength control algorithm which can be used to automatically determine the time steps for a stable, accurate and economic solution [10]. This paper presents a new automatic steplength control algorithm that is used with suitable recurrence schemes in solving such systems of ODEs. It also gives a discussion for the formulation of the problem, and the selection of the recurrence schemes.

\section{PROBLEM DEFINITION}

The energy equation which governs the heat transfer mechanism in metal cutting and other manufacturing processes is the conductive-transport equation in the form

$$
\begin{gathered}
\rho C\left(\frac{\partial T}{\partial t}+u \frac{\partial T}{\partial x}+v \frac{\partial T}{\partial y}+w \frac{\partial T}{\partial z}\right)=\frac{\partial}{\partial x}\left(k_{x} \frac{\partial T}{\partial x}\right) \\
+\frac{\partial}{\partial y}\left(k_{y} \frac{\partial T}{\partial y}\right)+\frac{\partial}{\partial z}\left(k_{z} \frac{\partial T}{\partial z}\right)+Q \text { on } \Omega,
\end{gathered}
$$

where $\rho$ is the density, $C$ is the specific heat, and $u$, $v$, and $w$ are the velocity components in $x, y$, and $z$ directions, respectively. Similarly, $k_{x}, k_{y}$, and $k_{z}$ are the thermal conductivity components corresponding to the principal axes $x, y$, and $z$, respectively. $T$ is the temperature and $Q$ the rate of heat generation.

Equation (1) is applied to the process domain $(\Omega)$ and subject to the boundary conditions describing the heat flow or temperature situation on the boundary of the domain. The part of the boundary on which the heat flows by conduction or convection is referred to as 'Cauchy' boundary conditions $\left(\Gamma_{2}\right)$ and is represented mathematically as

$$
\begin{aligned}
& k_{x} \frac{\partial T}{\partial x} n_{x}+k_{y} \frac{\partial T}{\partial y} n_{y}+k_{z} \frac{\partial T}{\partial z} n_{z} \pm q \\
& \pm h\left(T-T_{r}\right)=0 \text { on } \Gamma_{2} \subset \Gamma,
\end{aligned}
$$

where $n_{x}, n_{y}$, and $n_{z}$ are components of the normal vector, $h$ is the total heat transfer coefficient, and $T_{\text {r }}$ is the temperature of the environment.

The other boundary condition, where the temperature is known, is referred to as Dirichlet boundary conditions or specified temperature boundary $\left(\Gamma_{1}\right)$ and is represented mathematically as

$$
T=T(x, y, z, t) \text { on } \Gamma_{1} \subset \Gamma, t>0 .
$$

The temperature distribution in the domain $(\Omega)$ can be obtained from the solution of eqn (1) subject to the initial condition at $t=0$. It is mathematically represented by

$$
T=T_{0}(x, y, z, t) \text { on } \Omega, t=0 .
$$

This is a time-dependent heat flow problem which can be solved to obtain the temperature distribution at 
different time intervals. The analytical solution of this problem is complex. Therefore, the finite element method has been employed.

Finite element formulation approaches, such as weighted residuals with Galerkin's criterion or other formulations $[11,12]$, are used to derive a general element equation for the governing energy equation [eqn (1)] and the applicable boundary conditions [eqns (2) and (3)]. Then, the element equations can be explicitly evaluated for a particular element shape and an appropriate set of interpolation functions. Following the usual global assembly method, a system of first-order ordinary differential equations is obtained in the following form

$$
[C]\{\dot{T}\}+[K]\{T\}+\{F\}=0,
$$

where $T(x, y, z, t)$ denotes the field variable, $[K]$ is the convective-transport element matrix, $[C]$ is the heat capacitance matrix, and $\{F\}$ is the global load vector. In order to obtain the temperature distribution, this system of ODEs is to be solved using a suitable recurrence scheme subjected to initial conditions such as eqn (4). At this point it is important to mention that:

(a) the coefficients of the $[K]$ matrix in eqn (5) are non-symmetrical because of the first-order derivatives (the convective-transport part) in eqn (1);

(b) the system of ODEs [eqn (5)] is found to be 'stiff' (the ratio of the highest to lowest eigenvalues is very high) which necessitates very small steplengths at the initial phase of the numerical solution in order to avoid oscillatory results;

(c) the finite element solution of the steady state convective-transport problem gives oscillatory results if the mesh size exceeds a certain critical value [13], which may not be avoidable in many applications in manufacturing.

In order to avoid the oscillatory results during the steady-state solution [problem stated in (c)] an 'upwind' finite element scheme was adopted [13]. However, in order to avoid the problem stated above in (b) and (c) a suitable recurrence scheme and an automatic steplength control algorithm are needed to maintain the stability of the numerical solution.

\section{RECURRENCE SCHEME FOR SOLVING ODEs}

A recurrence scheme can be used to find a solution for eqn (5) in the time interval $\left(0, t_{0}\right)$, at times $t=t_{1}$, $t_{2}, \ldots, t_{n}, 0<t_{1}<t_{2}, \ldots, t_{m}=t_{0}$. Such a scheme transforms the system of ODEs into a system of algebraic equations of the following form

$$
[H]\{T\}_{n+1}=\{b\}, \quad n=0,1,2, \ldots, m-1
$$

in order to maintain the sparse (banded) characteristic matrices. Here $[H]$ is the characteristic matrix of the scheme [8]. There are several recurrence schemes which can be employed such as two-point, threepoint, and four-point recurrence schemes. High order recurrence schemes are more accurate but at the same time they require large memory computers and long computation times. In this work, a two-point recurrence scheme has been selected. It can be derived based on finite difference formulation [8] or finite element formulation [14]. The general form of the two-point recurrence scheme derived based on finite element formulation using the weighted residuals methods, takes the form

$$
\begin{gathered}
\left(\frac{1}{\Delta t}[C]+\theta[K]\right)\{T\}_{n+1} \\
+\left(-\frac{1}{\Delta t}[C]+(1-\theta)[K]\right)\{T\}_{n} \\
+\left((1-\theta)\{F\}_{n}+\theta\{F\}_{n+1}\right)=0, \\
n=0,1,2, \ldots, m-1 .
\end{gathered}
$$

Denoting

$$
[H]=\frac{1}{\Delta t}[C]+\theta[K]
$$

and

$$
\begin{aligned}
\{b\}=\left(\frac{1}{\Delta t}[C]-\right. & (1-\theta)[K])\{T\}_{n} \\
& -\left((1-\theta)\{F\}_{n}+\theta\{F\}_{n+1}\right)
\end{aligned}
$$

which yields eqn (6). This recurrence scheme is called $\theta$-method. setting $\theta=0$ in eqn (7) yields the Euler forward difference scheme, while $\theta=1 / 2$ gives the Crank-Nicholson-Galerkin (trapezoidal rule) representation and $\theta=1.0$ yields the Euler backward difference scheme.

The stability conditions for the solution of such a system of ODEs using the above recurrence scheme have been studied by several investigators [8-14]. These studies concluded that the use of $1 / 2<\theta<1$ is recommended to obtain a numerically stable solution. However, the use of $\theta=7 / 8$ is found to be optimal [13]. The stability of the solution of the system of ODEs requires a small time steplength in the initial phase of the numerical solution. This restriction can be overcome by employing a time step control scheme to control the step size and solution stability. Therefore, there is a need for a steplength control algorithm which can automatically determine the time step for stable, accurate and economical solution. In the following section, an algorithm has been derived to automatically control the step length for the implicit two-point recurrence schemes $(\theta>1 / 2)$. 


\section{STEPLENGTH CONTROL ALGORITHM}

In general, the strategy of controlling the steplength for certain recurrence scheme is to choose the largest possible steplength $(\Delta t)$ for which the local truncation error $(E T)$ remains upper bounded by a certain specified maximum allowable error [9]. The local truncation error for the linear multistep recurrence schemes can be expressed in the form

$$
E T=D_{\delta} T^{(\delta+1)}(\tau)(\Delta t)^{\delta+1}+O(\Delta t)^{\delta+1},
$$

where $\delta$ is the scheme order, $T^{(\delta+1)}(\tau)$ denotes the $(\delta+1)$ th derivatives of the solution $T(t)$ at $t=\tau$ and $t_{n}<\tau<{ }_{n+1}$, and $D_{\delta}$ is the error constant that depends on the order of the scheme. The values of $D_{\delta}, \delta$ and error formulae are given in Table 1 for the two-point recurrence schemes $(\theta$-method) of eqn (7). Assuming that the allowable truncation error per step is $E$, then for a steplength $\Delta t$ used with firstorder schemes, we have

$$
|E T|=\left|D_{\delta} T^{(2)}(\tau)(\Delta t)^{2}\right|<E .
$$

Let the steplength be changed to $\Delta t_{1}=\alpha \Delta t$, then

$$
\begin{aligned}
\left|E T_{1}\right| & =\left|D_{\delta} T^{(2)}(\tau)\left(\Delta t_{1}\right)^{2}\right|=\left|D_{\delta} T^{(2)}(\tau)(\alpha \Delta t)^{2}\right| \\
& =\alpha^{2}|E T|<E
\end{aligned}
$$

and $\alpha$ can be determined from the relation

$$
\alpha=\int\left(\frac{E}{S F|E T|}\right)
$$

where $S F$ is taken as a factor of safety, which can be determined experimentally to keep the error below
Table 1. Error formulae for $\theta$-scheme

\begin{tabular}{cccc}
\hline$\theta$ & $\delta$ & $D_{\delta}$ & $E T$ \\
\hline$\frac{1}{2}$ & 2 & $-\frac{1}{12}$ & $-\frac{1}{12} T^{(3)}(\tau) \Delta t^{3}$ \\
$\theta>\frac{1}{2}$ & 1 & $\frac{1}{2}-\theta$ & $\left(\frac{1}{2}-\theta\right) T^{(2)}(\tau) \Delta t^{2}$ \\
\hline
\end{tabular}

the limits. Once $E T$ is determined for the $n$th step, the value of $\alpha$ and the new steplength can be approximated using the backward difference formula as follows:

$$
T^{\prime}\left(t_{n}-\Delta t\right)=T^{\prime}(t)-\Delta t T^{\prime \prime}(t)+O(\Delta t) .
$$

Hence,

$$
T_{n}^{\prime \prime}=\frac{T_{n}^{\prime}-T_{n-1}^{\prime}}{\Delta t_{n}} .
$$

Similarly, $T_{n}^{\prime}$ and $T_{n-1}^{\prime}$ can be determined from the following equations

$$
\begin{gathered}
T_{n}^{\prime}=\frac{T_{n}-T_{n-1}}{\Delta t_{n}} \\
T_{n-1}^{\prime}=\frac{T_{n-1}-T_{n-2}}{\Delta t_{n-1}} .
\end{gathered}
$$

Substituting (14) and (15) in (13), we get

$$
T_{n}^{\prime \prime}=\frac{T_{n}-\left(1+\alpha_{n}\right) T_{n-1}+\alpha_{n} T_{n-2}}{\Delta t_{n}^{2}}
$$

If we consider $T_{n}^{\prime \prime}(\tau)=T_{n}^{\prime \prime}$, i.e., taking $\tau=t_{n}$, then we can write the formula to estimate $E T$, for the $n$th time step, for the two-point recurrence schemes of $\theta>1 / 2$ as follows:

$$
E T=\left(\frac{1}{2}-\theta\right)\left(T_{n}-\left(1+\alpha_{n}\right) T_{n-1}+\alpha_{n} T_{n-2}\right) .
$$

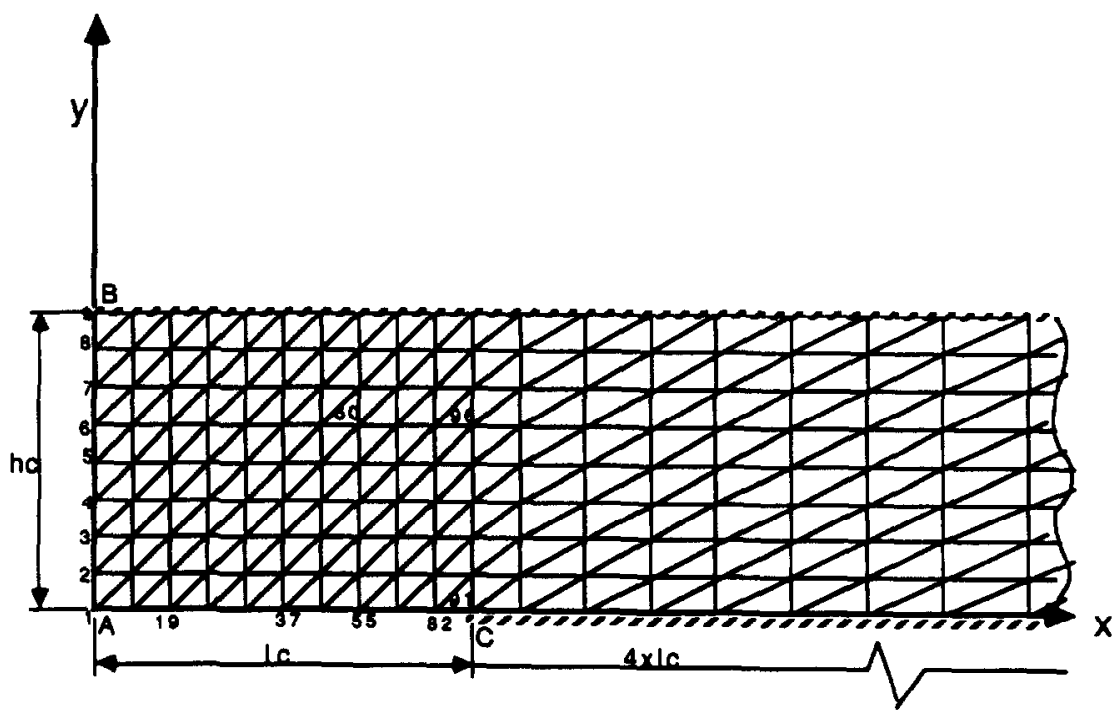

Fig. 1. Mesh and thermal boundaries. 
Table 2. Boundary data and properties of the chip material

\begin{tabular}{lcl}
\hline Boundary data & & \\
Shear plane temperature $\left(T_{s}\right)$ & 703.91 & $\mathrm{~K}$ \\
Strength of friction heat source $\left(q_{f}\right)$ & 42.94 & $\mathrm{~W} / \mathrm{mm}^{2}$ \\
Heat transfer coefficient $(h)$ & 0.00248 & $\mathrm{~W} / \mathrm{mm}^{2} \mathrm{~K}$ \\
Properties of the chip material (carbon steel grade 1035$)$ & \\
Thermal conductivity $(K)$ & 0.052338 & $\mathrm{~W} / \mathrm{mm} \mathrm{K}$ \\
Volumetric specific heat $(C)$ & 0.0034757 & $\mathrm{~J} / \mathrm{mm}^{3} \mathbf{K}$ \\
\hline
\end{tabular}

Estimating the error $E T$ at the starting point is not an easy task because three values of $T\left(T_{n}, T_{n-1}\right.$, and $T_{n-2}$ ) are required. Therefore, a starting routine should be established to begin the iteration. The starting procedure is to choose any steplength $\Delta t$ and move two steps, then estimale $E T$. If $|E T|<E$, determine the new steplength and the solution can be proceeded. But, if $|E T|>E$, determine $(\alpha)$ and a new starting steplength and repeat the starting steps until $|E T|$ becomes less than $E$.

For a system of equations, the error is equal to the $L_{2}$ Euclidean norm of the individual elements of $E T$, which is defined by

$$
\|E T\|_{L_{2}}=\sqrt{ }\left(\{E T\}^{T}\{E T\}\right)
$$

The relative error is usually used with 'stiff' system of ODEs instead of the absolute error. The relative error is defined as

$$
\|E T\|=\frac{\|E T\|}{\left\|T_{n}\right\|}=\sqrt{\left(\frac{\{E T\}^{T}\{E T\}}{\left\{T_{n}\right\}^{T}\left\{T_{n}\right\}}\right)},
$$

where $\{T\}_{n}$ are the values of the function at the $n$th step. The above algorithm has been implemented in the finite element program which has been developed for a three node triangular element and tested with $\theta=2 / 3,7 / 8$, and 1 . The next section provides a practical example for testing the above algorithm along with presentation of the results.

\section{TESTING THE ALGORITHM}

In order to test the algorithm, the chip flow in metal cutting has been selected since the transient convective-transport equation [eqn (1)] is applicable on the chip domain. The chip domain, together with the thermal boundaries, is shown in Fig. 1. The shear plane $(\mathrm{AB})$ temperature $\left(T_{s}\right)$ is taken as the average temperature of the shear plane heat source and considered as the prescribed temperature boundary, i.e.

$$
T(0, y)=T_{s}, \quad x=0,0<y<h_{c},
$$

where $h_{\mathrm{c}}$ is the chip thickness. The tool/chip interface zone (AC) is a conductive boundary which is assumed to be a plane heat source with uniform strength $\left(q_{f}\right)$, i.e.

$$
k\left(\frac{\partial T(x, 0)}{\partial x}+\frac{\partial T(x, 0)}{\partial y}\right)=q_{f}, \quad 0 \leqslant x \leqslant 1_{c} .
$$

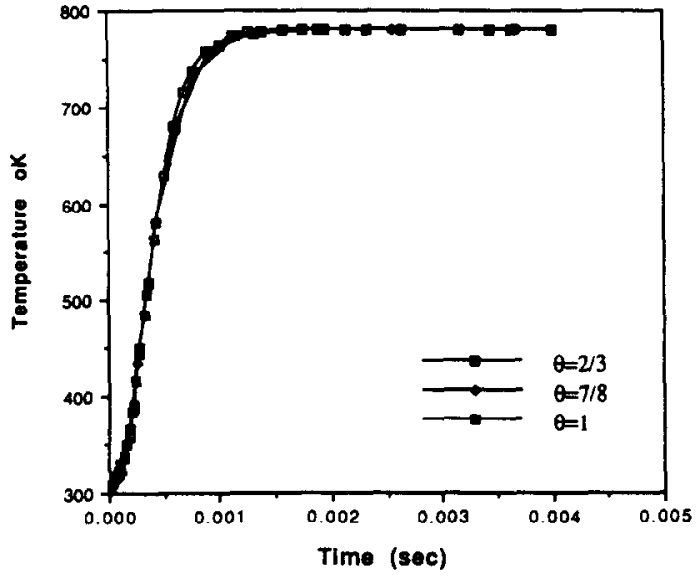

Fig. 2. Transient nodal temperature for node 37 .

At a distance far from the heat generation zone, the chip temperature is assumed to be uniform. Thus, no heat is conducted and the boundary is considered insulated. Similarly, for the remaining boundaries, the convection and radiation to air are neglected, and therefore, the chip is considered insulated, i.e.

$$
\begin{aligned}
\frac{\partial T(x, y, t)}{\partial n}=0, & l_{c} \leqslant x \leqslant 5 l_{c}, y=0 \\
& \text { and } 0 \leqslant x \leqslant 5 l_{c}, y=h_{c} .
\end{aligned}
$$

The boundary data and the properties of the chip material are given in Table 2. The initial condition is assumed to be the room temperature, i.e.

$$
T(x, y, 0)=293 \mathrm{~K} .
$$

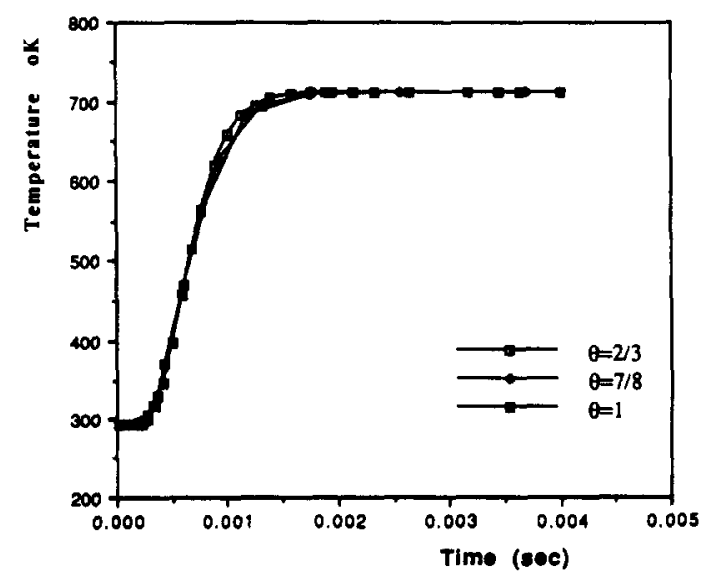

Fig. 3. Transient nodal temperature for Node 60 . 


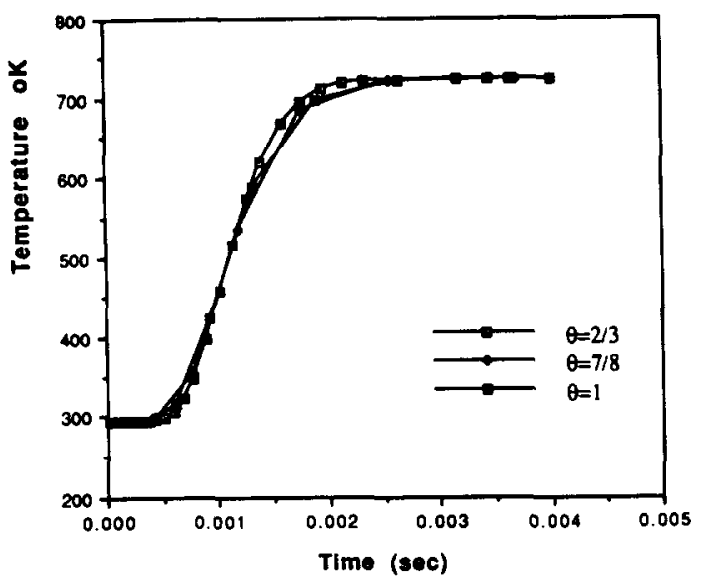

Fig. 4. Transient nodal temperature for Node 96.

The transient temperature distribution in the chip was obtained for three different values of $(\theta=2 / 3$, $7 / 8$, and 1 ), and a time span of $4 \mathrm{msec}$ where it is expected to reach steady-state. The truncation error is assumed to be bounded with $E=0.001$. The transient temperatures at time points with the solution using $\theta=2 / 3,7 / 8$, and 1 are plotted in Figs $2-4$ for node 37 , node 60 , and node 96 , respectively. It is seen that the instantaneous nodal temperatures obtained with the three values of $\theta$ coincide (the differences indicated on the graph are from the plotting routine where it connects the points with straight lines), and no oscillation has been detected. The time step is very small at the start and increases as the solution progresses. The number of time steps to reach a span of $4 \mathrm{msec}$ is 26,17 , and 22 for $\theta=2 / 3,7 / 8$, and 1 , respectively. This indicates that $\theta=7 / 8$ is optimal

Table 3. Steady-state nodal temperatures

\begin{tabular}{ccccc}
\hline Node & $\begin{array}{l}\text { Steady-state } \\
\text { formulation }\end{array}$ & $\theta=1$ & $\theta=7 / 8$ & $\theta=2 / 3$ \\
\hline 10 & 737.58859 & 737.5886 & 737.5886 & 737.5886 \\
37 & 780.03191 & 780.0319 & 780.0319 & 780.0319 \\
50 & 714.23343 & 714.2334 & 714.2334 & 713.2334 \\
56 & 766.89163 & 766.8916 & 766.8916 & 766.8916 \\
67 & 733.48651 & 733.4865 & 733.4865 & 733.4865 \\
80 & 709.41445 & 709.4144 & 709.4145 & 709.4145 \\
85 & 744.05193 & 744.0519 & 744.0519 & 744.0519 \\
82 & 820.396 & 820.3959 & 820.396 & 820.396 \\
91 & 810.75347 & 810.7533 & 810.7535 & 810.7535 \\
96 & 724.20741 & 724.2073 & 724.2074 & 724.2074 \\
120 & 762.53476 & 762.5316 & 762.5347 & 762.5347 \\
130 & 751.9697 & 751.962 & 751.9696 & 751.9695 \\
135 & 724.60535 & 724.5985 & 724.6053 & 724.6051 \\
137 & 761.52496 & 761.5038 & 761.5244 & 761.5246 \\
146 & 758.34968 & 758.3017 & 758.3585 & 758.348 \\
150 & 738.98989 & 738.9454 & 738.989 & $\mathbf{7 3 8 . 9 8 7 8}$ \\
152 & 731.62899 & 731.5868 & 731.6284 & $\mathbf{7 3 1 . 6 2 6 5}$ \\
157 & 749.07478 & 748.0438 & 749.068 & 749.0744 \\
160 & 736.46429 & 736.3306 & 736.4571 & $\mathbf{7 3 6 . 4 6 4 6}$ \\
166 & 748.26782 & 748.0204 & $\mathbf{7 4 8 . 2 5 5 9}$ & $\mathbf{7 4 8 . 2 6 2 9}$ \\
177 & 741.8936 & 741.1884 & 741.8364 & $\mathbf{7 4 1 . 9 2 2 7}$ \\
180 & 736.54605 & 736.8256 & 737.4881 & $\mathbf{7 3 7 . 5 8 2 3}$ \\
\hline
\end{tabular}

since it requires the least computational effort. This result is in agreement with that obtained by other investigators $[9,13]$.

The steady-state solution of the above problem, based on the finite element formulation for the steady convective-transport energy equation, has been obtained. The results obtained were, then, compared to the steady-state conditions for the three values of $\theta$. Table 3 shows the steady-state temperatures for selected nodes on the chip domain. The nodal temperatures obtained with the four solutions are exactly the same. Hence, it is concluded that the steplength control algorithm is successfully working and $\theta=7 / 8$ is optimal.

\section{REFERENCES}

1. E. Orady and M. S. Gadala, Finite element applications to selected manufacturing processes. Int. J. Sci. Technol. 2, 18-29 (1989).

2. G. Boothroyd, Temperature in orthogonal metal cutting. Proc. Inst. Mech. Engrs 177, 789-810 (1963).

3. A. S. Wifi and E. A. Orady, Finite element applications in manufacturing processes, an overview. Proc. of The Third Cairo University Conference on Mechanical Design and Production, Cairo, Egypt, Dec. 28-30, 1985. Current Advances in Mechanical Design and Production (Edited by S.E.A. Bayoumi and M. Y. A. Younan), pp. 417-32. Pergamon Press, Oxford (1986).

4. E. Friedman, Numerical simulation of gas tungsten-arc welding process. Numer. Modeling Manufacturing Proc. ASME PVP-PB-025, 35-47 (1977).

5. K. J. Trigger and B. T. Chao, Temperature distribution at the tool/chip interface in metal machining. Trans ASME 77, 1107-1121 (1955).

6. A. O. Tay, M. G. Stevenson and G. Davis, Using the finite element method to determine temperature distribution in orthogonal machining. Proc. Inst. Mech. Engrs 188, 682-638 (1974).

7. M. Zorzi and P. Mazzantini, Continuous casting solidification model by finite element method, In Numerical Methods in Industrial Forming Processes, pp. 401-411. Pineridge Press, Swansea (1982).

8. Z. Zlatev and P. G. Thomsen, Application of backward differentiation methods to the finite element solution of time dependent problems. Int. J. Numer. Meth. Engng 14, 1051-1061 (1979).

9. J. D. Lambert, Computational Methods in Ordinary Differential Equations. John Wiley (1973).

10. M. Zlamal, The finite element methods in heat conduction problems. In The Mathematics of Finite Element and Application II (Edited by J. Whiteman), pp. 85-104. Academic Press.

11. O. C. Zienkiewicz, The Finite Element Method, 3rd Edn. McGraw-Hill (1977).

12. O. C. Zienkiewicz and C. J. Parekh, Transient field problem: two-dimensional and three-dimensional analysis by isoparametric finite elements. Int. J. Numer. Meth. Engng 2, 61-71 (1970).

13. P. S. Heinrich, P. S. Huyakorn and O. C. Zienkiewicz, An 'Upwind' finite element scheme for two dimensional convective-transport equation. Int. J. Numer. Meth. Engng VII, 131-143 (1977).

14. O. C. Zienkiewicz and R. W. Lewis, An analysis of various time-stepping schemes for initial value problems. Earthquake Engng Struct. Dyn. 1, 407-408 (1973). 